\title{
Hemorragia de intestino delgado
}

\author{
Araceli Muñoz-Bautista* \\ Departamento de Endoscopia Gastrointestinal, Hospital de Especialidades, Centro Médico Nacional La Raza, Ciudad de México, Méx., México
}

\section{Resumen}

La hemorragia del intestino delgado representa del 5 al 10\% de todas las hemorragias gastrointestinales. A pesar de los avances en imagen, endoscopia y técnicas terapéuticas mínimamente invasivas, su diagnóstico y tratamiento sigue siendo un desafío y un algoritmo estandarizado para abordar la sospecha de hemorragia del intestino delgado sigue siendo difícil de alcanzar. Se presenta un análisis de los trabajos presentados en Semana de Gastroenterología de la Unión Europea 2020 (UEGW) y la Semana de Enfermedades Digestivas de los EEUU 2021 (DDW) llevadas a cabo en modalidad virtual en relación con hemorragia de intestino medio.

Palabras clave: Hemorragia. Intestino medio. Angiodisplasias.

\section{¿Cuándo está indicada la enteroscopia?}

La enteroscopia asistida por dispositivo complementa a la cápsula endoscópica en muchos casos de hemorragia de intestino delgado, suele ser la siguiente herramienta diagnóstica y terapéutica. Se recomienda que la cápsula endoscópica y la enteroscopia se realicen lo más cerca posible del episodio hemorrágico para mejorar el rendimiento diagnóstico ${ }^{\dagger}$. Sin embargo, no hay datos claros sobre el cuál es el mejor momento para realizar la enteroscopia. Por lo que un estudio evalúa si el intervalo más corto entre la cápsula endoscópica y un segundo procedimiento endoscópico aumenta el rendimiento del procedimiento secundario. Se evaluaron 303 pacientes a los que se les realizó cápsula endoscópica entre enero de 2018 y mayo de 2020 con diagnóstico de hemorragia gastrointestinal o anemia de etiología desconocida. En 237 pacientes (78\%) el resultado fue negativo y en 66 pacientes (22\%) se identificó hemorragia activa. A $56 / 66$ pacientes ( $85 \%$ ) se les realizó un procedimiento endoscópico (endoscopia superior/enteroscopia de empuje/doble balón) en los siguientes 90 días. En 22/56 (39\%) se identificó hemorragia activa y en $34 / 56(61 \%)$ no se identificó hemorragia activa. Se encontró que el tiempo promedio entre la cápsula endoscópica y el procedimiento endoscópico posterior a esta en los pacientes que tuvieron hemorragia activa fue significativamente más corto (2.23 días) que en los casos donde no fue identificada hemorragia activa (5.21 días; $p=0.031$ ). Por lo que se concluye que acortar el periodo de tiempo entre estos dos estudios incrementa la probabilidad de encontrar hemorragia activa y de tratamiento endoscópico exitoso ${ }^{2}$. Otro trabajo evalúa si la enteroscopia asistida con balón (EAB) realizada de forma urgente afecta las tasas de resangrado, el tiempo de resangrado y la mortalidad en pacientes con hemorragia digestiva de origen oscuro manifiesta (HDOOM). Fue un estudio de cohorte retrospectivo, que incluyó a pacientes sometidos a EAB con diagnóstico HDOOM entre 2010-2019, los pacientes se distribuyeron en 2 grupos: 1) EAB urgente y 2) EAB no urgente. Se incluyeron 54 pacientes; 17 (31.5\%) fueron sometidos a EAB en las primeras $72 \mathrm{~h}$. El rendimiento diagnóstico $(\mathrm{RD})$ y el rendimiento terapéutico $(\mathrm{RT})$ de $E A B$ urgente fue (RD: 88.2\%; $n=15 ; R T: 94.1 \% ; n=16$ ) mayor en comparación con $E A B$ no urgente (RD: $59.5 \%$; $n=22 ; R T: 45.9 \% ; n=17)(R D p=0.03 ; R T p=0.001)$. 
La tasa de resangrado a 1, 2 y 5 años fue del 32, 34 y $37 \%$ respectivamente. El resangrado fue menor después de $E A B$ urgente $(17.6 \% ; n=3)$ en comparación a $E A B$ no urgente $(45.9 \% ; n=17)(p=0.04)$. El resangrado se presentó antes en $E A B$ no urgentes, siendo a los 6 meses (32.5\%) y a los 36 meses $(41.3 \%)(p=0.05)$. La mortalidad relacionada con la HDOOM fue del 8.1\% $(n=3)$ para las $E A B$ no urgentes y del $0 \%$ para los $E A B$ urgentes $(p=0.5)$. Este estudio concluye que la $E A B$ urgente se asocia con mayor RD y RT, con menor resangrado y tendencia hacia un mayor tiempo libre de resangrado ${ }^{3}$.

\section{Hemorragia refractaria del intestino delgado}

Actualmente se sabe que el resangrado se presenta en hasta el $45 \%$ de los pacientes 2-3 años después de la intervención endoscópica ${ }^{4}$. Silva, et al. nos presentan un trabajo sobre la predicción del resangrado posterior a realizar una cápsula endoscópica en relación con la puntuación de RHEMITT (validación externa) (Tabla 1). Fue un estudio de cohorte retrospectivo, realizado entre enero de 2017 y diciembre de 2018. Se calculó la puntuación RHEMITT y posteriormente se evaluó la precisión de la puntuación para la predicción de resangrado, incluyeron 160 pacientes, el tiempo medio de seguimiento fue de 20 meses. Se produjo resangrado en el $14.4 \%(n=23)$. El resangrado a los $6,12,18$ y 24 meses fue del $6.3,12,14.2$ y $15.5 \%$ respectivamente. Hubo una asociación significativa entre la puntuación RHEMITT y resangrado $(p<0.001)$. El resangrado fue presentado antes en pacientes de riesgo intermedio y alto, siendo a los 6 meses $13.6 \%$ y a los 24 meses 28.4\% ( $p<0.01)$. Este estudio realizado en una cohorte de validación externa confirma la utilidad y precisión del puntaje RHEMITT para predecir resangrado posterior a la cápsula endoscópica ${ }^{5}$.

Se ha reportado que las angiodisplasia se localizan en un $57-80 \%$ en intestino delgado ${ }^{6}$ y son las causantes del $50-60 \%$ de la hemorragia gastrointestinal de origen oscuro diagnosticada por cápsula endoscópica, debido a la alta tasa de episodios de resangrado que se reporta en hasta el $70 \% 7$.

Por lo que el estudio de García-Compean, et al. desarrolla, evalúa y valida una nueva puntuación para medir la severidad de las angiodisplasias en intestino delgado por cápsula endoscópica denominada CESBAI (Tabla 2). Se analizaron 22 vídeos por 4 observadores con evaluaciones cegadas e independientes. Los hallazgos de los cuatro observadores no tuvieron
Tabla 1. Predicción del resangado posterior a realizar una cápsula endoscópica en relación con la puntuación de RHEMITT*

\begin{tabular}{|c|c|c|c|}
\hline & & & Puntuación \\
\hline $\mathrm{R}$ & Renal disease & $\begin{array}{l}\text { Enfermedad renal } \\
\text { crónica }\end{array}$ & 3 \\
\hline $\mathrm{H}$ & Heart failure & Insuficiencia cardiaca & 1 \\
\hline$E$ & $\begin{array}{l}\text { Endoscopic } \\
\text { capsule lesions }\end{array}$ & $\begin{array}{l}\text { Lesiones P1 } \\
\text { (clasificación de } \\
\text { Saurin) } \\
\text { Lesiones P2 }\end{array}$ & $\begin{array}{l}2 \\
3\end{array}$ \\
\hline M & Major bleeding & Sangrado mayor & 5 \\
\hline I & $\begin{array}{l}\text { Incomplete } \\
\text { capsule }\end{array}$ & Cápsula incompleta & 2 \\
\hline $\mathrm{T}$ & Tobacco & Tabaquismo & 2 \\
\hline $\mathrm{T}$ & $\begin{array}{l}\text { Treatment by } \\
\text { endoscopy }\end{array}$ & $\begin{array}{l}\text { Tratamiento } \\
\text { endoscópico }\end{array}$ & 2 \\
\hline
\end{tabular}

*Riesgo bajo: 0-3 puntos. Riesgo intermedio: 4-10 puntos. Riesgo bajo: 11-18 puntos.

Adaptada de Silva, et al., $2020^{4}$.

Tabla 2. CESBAl: puntuación para medir la severidad de las angiodisplasias en intestino delgado por cápsula endoscópica*

\begin{tabular}{|c|c|c|c|}
\hline \multirow[t]{2}{*}{$\begin{array}{l}\text { A. Extensión de } \\
\text { las lesiones }\end{array}$} & E1 & $\begin{array}{l}\text { Localizada en la primera } \\
\text { mitad del intestino } \\
\text { delgado }\end{array}$ & 1 punto \\
\hline & E2 & $\begin{array}{l}\text { Distribuidas en todo el } \\
\text { intestino delgado }\end{array}$ & 2 puntos \\
\hline \multirow{3}{*}{$\begin{array}{l}\text { B. Número de } \\
\text { lesiones }\end{array}$} & N1 & $<5$ lesiones & 1 punto \\
\hline & N2 & 5-10 lesiones & 2 puntos \\
\hline & N3 & $>10$ lesiones & 3 puntos \\
\hline \multirow{4}{*}{$\begin{array}{l}\text { C. Probabilidad } \\
\text { de asociación } \\
\text { con hemorragia }\end{array}$} & P1 & Manchas rojo pálido & 1 punto \\
\hline & P2 & Manchas rojas brillantes & 2 puntos \\
\hline & P3 & $\begin{array}{l}\text { Lesión con estigmas de } \\
\text { sangrado (úlcera, } \\
\text { coágulo o restos } \\
\text { hemáticos) }\end{array}$ & 3 puntos \\
\hline & P4 & $\begin{array}{l}\text { Lesión con sangrado } \\
\text { activo }\end{array}$ & 4 puntos \\
\hline
\end{tabular}

*Puntuación 6-20 puntos. Fórmula: Ex1 + Nx2 + Px3.

diferencias estadísticamente significativas. La puntuación CESBAI obtenida por cada observador tuvo un acuerdo estadísticamente significativo entre estos $(p<0.05)$. Los autores concluyen que el uso de 
CESBAI demostró ser una puntuación endoscópica confiable y reproducible para la evacuación de la angiodisplasias en intestino delgado y fácil de utilizar, sin embargo, amerita validación en otros estudios con mayor población antes de evaluar su poder para predecir la recurrencia del sangrado ${ }^{8}$.

Actualmente se sugiere que los anticoagulantes y/o la terapia antiplaquetaria sea descontinuada si es posible en pacientes con hemorragia de intestino delgado, ya que incrementa el hallazgo positivo en pacientes a los que se les realiza cápsula endoscópica ${ }^{9}$. Se presenta un estudio que evalúa la relación entre la terapia antitrombótica y el rendimiento de la CE, así como la necesidad de intervenciones endoscópicas del intestino delgado y compara el uso de antiplaquetarios y anticoagulantes. Se realizó en un centro de tercer nivel, se incluyeron a los pacientes a los que se les realizó CE de enero de 2018 a mayo de 2020. Se realizaron 311 estudios de CE, el promedio de edad fue de 70 años, 115 (37\%) no utilizaba terapia antitrombótica, 99 (32\%) usó terapia antiplaquetaria, $36(12 \%)$ tenían tratamiento con anticoagulantes y $61(20 \%)$ utilizaron terapia antiplaquetaria y anticoagulante. El uso de antiplaquetarios fue asociado con una alta probabilidad de hallazgos positivos en la cápsula endoscópica $(74 \% ; p=0.030)$, también hubo una alta probabilidad de realizarles enteroscopia y de tratamiento endoscópico dentro de los siguientes 90 días (36\%; $p=0.045)$. La terapia con anticoagulantes y la terapia combinada no predijeron visualización de sangrado activo o probabilidad de intervención endoscópica ( $p=0-97)$. Sin embargo, en el centro tienen la practica estándar de suspender los anticoagulantes y mantener la terapia antiplaquetaria en los pacientes que ingresan, por lo que en futuros estudios es necesario aclarar el tiempo de uso de anticoagulantes, la dosis y perfil de tiempos de coagulación previos al estudio de la CE.

\section{Terapia farmacológica en hemorragia de intestino delgado}

El manejo médico actualmente tiene un papel limitado en la hemorragia de intestino delgado. Los análogos de la somatostatina (AS), como octreótida, parecen disminuir las tasas de resangrado y la necesidad de transfusión de concentrados eritrocitarios (CE) en las angiodisplasias gastrointestinales $(\mathrm{AGI})^{10}$. Debido a la falta de estudios para evaluar su eficacia Goltstein, et al. presentan un metaanálisis para establecer la eficacia del tratamiento con AS sobre la necesidad de transfusiones sanguíneas en pacientes con angiodisplasias. Se analizaron los datos de 212 pacientes de 11 estudios (edad media: 71 años, 53\% hombres). Se clasificaron como buenos respondedores a los que presentaban (reducción $\geq 50 \%$ ) o malos respondedores $(<50 \%)$ de las transfusiones. Los pacientes experimentaron una disminución media de transfusiones de CE de 13.17 a 3.53 en un periodo medio de 12.2 meses. La disminución media de la transfusión de CE fue del $73.2 \%$ (IC 95\%: $70.9-75.4 \%$; $p<0.0001$ ). Se observó una buena respuesta en 177/212 pacientes $(83.5 \%)$ y $35 / 212$ pacientes (16.5\%) tuvieron una mala respuesta. La mayoría de los pacientes que respondieron bien (109/177; 61.6\%) no necesitaron transfusión de CE, durante el tratamiento con AS. Los eventos adversos ocurrieron en $38 / 212$ pacientes (17.9\%). Los más comunes fueron heces blandas (3.3\%), colelitiasis $(2.4 \%)$, flatulencia $(1.9 \%)$ y dolor abdominal (1.4\%). Solo 10/212 pacientes $(4.7 \%)$ interrumpieron el tratamiento. Este estudio concluye que el tratamiento con AS es eficaz y seguro en la mayoría de los pacientes dependientes de transfusiones sanguíneas por hemorragia secundaria a angiodisplasia gastrointestinal ${ }^{11}$.

\section{Financiamiento}

La presente investigación no ha recibido ayudas específicas provenientes de agencias del sector público, sector comercial o entidades sin ánimo de lucro.

\section{Conflicto de intereses}

Los autores declaran no tener conflicto de intereses.

\section{Bibliografía}

1. Shinozaki S, Yamamoto H, Yano T, Sunada K, Miyata T, Hayashi Y, et al. Long-term outcome of patients with obscure gastrointestinal bleeding investigated by double-balloon endoscopy. Clin Gastroenterol Hepatol. 2010;8:151-8.

2. Boortalary T, Au C, Shinn B, Infantolin A, Tofain C. ID: 3524100 Shorter interval from video capsule endoscopy to second endoscopy increases yeld of secondary procedure. Gastrointest Endosc [Internet]. 2021;93(6 Suppl):AB354-AB355. Disponible en: https://www.giejournal. org/article/S0016-5107(21)01142-1/fulltext

3. Silva JC, Pinho R, Ponte A, Rodrigues A, Rodrigues J, Gomes AC, et al. Does urgent balloon assisted enteroscopy impacts rebleeding and mortality in overt obscure gastrointestinal bleeding? Scand J Gastroenterol. 2020;55(10):1243-7.

4. Jackson CS, Gerson LB. Management of gastrointestinal angiodysplastic lesions (GIADs): a systematic review and meta-analysis. Am J Gastroenterol. 2014:109:474-83, quiz 484.

5. Silva JC, Pinho R, Ponte A, Rodrigues A, Ribeiro Gomes AC, Afeto E, et al. Predicting the risk of rebleding after capsule endoscopy in obscure gastrointestinal bleeding - External validation of the RHEMITT score. Dig Dis. 2020 Jul 8. doi: 10.1159/000509986. Online ahead of print

6. Bollinger E, Raines D, Saitta P. Distribution of bleeding gastrointestinal angioectasias in a Western population. World J Gastroenterol. 2012;18:6235-9.

7. García-Compeán D, Del Cueto-Aguilera ÁN, Jiménez-Rodríguez AR, González-González JA, Maldonado-Garza HJ. Diagnostic and therapeutic challenges of gastrointestinal angiodysplasias: A critical review and view points. World J Gastroenterol. 2019;25(21):2549-64. 
Endoscopia. 2021;33(Supl 1)

8. García-Compeán D, del Cueto-Aguilera ÁN, Ángel N, Borjas-Alamaguer, Jiménez-Rodríguez AR, Muñoz-Ayala JM, et al. Evaluation and validation of a new method for measuring the severity of the small bowel angiodysplasias (SBAD) by video capsule endoscopy (CESBAI). Dig Dis 2021 Mar 29. doi: 10.1159/000516163. Online ahead of print

9. Gerson LB, Fidler JL, Cave DR, Leighton JA. Clinical guideline: Diagnosis and management of small bowel bleeding. Am J Gastroenterol. 2015;110(9):1265-87;quiz 1288.
10. Jackson CS, Gerson LB. Management of gastrointestinal angiodysplastic lesions (GIADs): a systematic review and meta-analysis. Am J Gastroenterol. 2014;109:474-83;quiz 484.

11. Goltstein LCMJ, Grooteman KV, Rocco A, Holleran G et al Somatostatin analogues are effective and safe in transfusion dependent gastrointestinal bleeding due to angiodysplasia: an individual patient data meta-analysis. Presentación en Póster (Small intestinal). United European Gastroenterology UEG Week Virtual 2020, Virtual. P0290. 